\title{
Lumbar computed tomography scans are not appropriate surrogates for bone mineral density scans in primary adult spinal deformity
}

\author{
Eitan M. Kohan, MD, ${ }^{1}$ Venu M. Nemani, MD, PhD, ${ }^{2}$ Stuart Hershman, MD, ${ }^{3}$ Daniel G. Kang, MD, ${ }^{4}$ and \\ Michael P. Kelly, MD, MSc ${ }^{1}$
}

${ }^{1}$ Department of Orthopedic Surgery, Washington University in St. Louis, Missouri; ${ }^{2}$ Raleigh Orthopaedic Clinic, Raleigh, North Carolina; ${ }^{3}$ Department of Orthopaedic Surgery, Massachusetts General Hospital, Boston, Massachusetts; and ${ }^{4}$ Madigan Army Medical Center, Tacoma, Washington

\begin{abstract}
OBJECTIVE The authors examined the correlation between lumbar spine CT Hounsfield unit $(\mathrm{HU})$ measurements and bone mineral density measurements in an adult spinal deformity (ASD) population.

METHODS Patients with ASD were identified in the records of a single institution. Lumbar CT scans were reviewed, and the mean HU measurements from L1-4 were recorded. Bone mineral density (BMD) was assessed using femoral neck and lumbar spine dual-energy $x$-ray absorptiometry (DEXA). The number of patients who met criteria for osteoporosis was determined for each imaging modality.
\end{abstract}

RESULTS Forty-eight patients underwent both preoperative DEXA and CT scanning. Forty-three patients were female and 5 were male. Forty-seven patients were Caucasian and one was African American. The mean age of the patients was 62.1 years. Femoral neck DEXA was more likely to identify osteopenia $(n=26)$ than lumbar spine DEXA $(n=8)$ or lumbar CT HU measurements $(n=6)(p<0.001)$. There was a low-moderate correlation between lumbar spine CT and lumbar spine DEXA $(r=0.463, p<0.001)$, and there was poor correlation between lumbar spine CT and femoral neck $\operatorname{DEXA}(r=0.303, p=0.036)$.

CONCLUSIONS Despite the opportunistic utility of lumbar spine CT HU measurements in identifying osteoporosis in patients undergoing single-level fusion, these measurements were not useful in this cohort of ASD patients. The correlation between femoral neck DEXA and HU measurements was poor. DEXA assessment of BMD in ASD patients is essential to optimize the care of these complicated cases.

https://thejns.org/doi/abs/10.3171/2017.9.FOCUS17476

KEY WORDS adult spinal deformity; Hounsfield units; bone mineral density; computed tomography; osteoporosis

$\mathrm{B}$ Отн оsteoporosis and adult spinal deformity (ASD) are associated with advancing age and often occur concurrently. ${ }^{14}$ Osteoporosis is a common disease in the United States, affecting an estimated 53.6 million Americans in 2010..$^{39}$ While the exact prevalence of ASD is unknown, estimates range from $2.5 \%$ to $25 \%$ of the population. ${ }^{27,13,24,27,31}$ Preoperative identification and treatment of patients with osteoporosis are important, as accurate measurement of BMD predicts postoperative instability and fracture risk. ${ }^{17,21}$ However, the most widely used modality with which to diagnose osteoporosis, lumbar dual-energy x-ray absorptiometry (DEXA), often overesti- mates BMD in patients with scoliosis and/or degenerative changes. ${ }^{5}$ This overestimation is related to the magnitude of the spinal curve, rotation of the spine, the presence of osteophytes, facet joint osteoarthritis, bone sclerosis, and disc space narrowing. ${ }^{10,16,18,23,25,26,28,29}$ Overestimation of the BMD in patients with scoliosis can lead to late diagnosis of osteoporosis, resulting in missed opportunities to intervene and optimize the bone health of these patients preoperatively. Current practices use femoral neck DEXA scans to more accurately measure BMD and guide therapy in this population.

Hounsfield unit (HU) measurements obtained from CT

ABBREVIATIONS ASD = adult spinal deformity; $B M D$ = bone mineral density; $D E X A=$ dual-energy $x$-ray absorptiometry; $H U$ = Hounsfield unit. SUBMITTED July 25, 2017. ACCEPTED September 20, 2017. INCLUDE WHEN CITING DOI: 10.3171/2017.9.FOCUS17476. 
scans correlate to lumbar spine BMD in cases of spinal trauma and vertebral compression fractures. ${ }^{33}$ This modality allows for assessment of osteoporosis and fracture risk without the need for further imaging via DEXA. In degenerative lumbar spine surgery, higher HU measurements are associated with lower rates of pseudarthrosis. ${ }^{34}$ HU measurements may also help identify osteoporosis in patients in whom the diagnosis was not established by DEXA or other modalities. ${ }^{38}$ Identification of osteoporosis is essential in ASD surgery, to mitigate both the risks associated with the surgery and the risks of untreated osteoporosis in general. . $^{1,6,8,37}$

The relationship between lumbar CT HUs in ASD and DEXA-measured BMD is unknown. The purpose of this study was to evaluate the relationship between lumbar HUs and BMD in a cohort of ASD patients. These results may provide data immediately available to the physician to begin preoperative optimization and to aid the shared decision-making process in ASD.

\section{Methods \\ Patient Population}

After institutional review board approval was obtained, we reviewed the surgical records of a single center to identify primary ASD patients treated from 2009 to 2014. Inclusion criteria included having both lumbar CT scans and femoral neck DEXA images. Exclusion criteria included incomplete medical records, lumbar spinal instrumentation, prior cement augmentation, and previous spinal fusion.

\section{Data Collection}

All data were collected on standardized forms. Demographic data collected included age, sex, and race. DEXA records were reviewed and T-scores and BMD values (measured in $\mathrm{g} / \mathrm{cm}^{2}$ ) in the lumbar spine and femoral neck were recorded. CT scans were reviewed using Merge RadSuite version 8.30 (Merge Healthcare). To obtain the HU values, lumbar CT scans were reviewed according to the method of Schreiber et al. ${ }^{33}$ These were acquired at 4 lumbar vertebrae (L-1, L-2, L-3, and L-4) for each CT scan. An elliptical region confined to the medullary canal of the vertebral body was used in 3 distinct axial cuts in each vertebra: immediately inferior to the superior endplate, in the middle of the vertebral body, and immediately superior to the inferior endplate. The average of these 3 values was used to define the mean HU value of the vertebra, and the mean of each vertebra was averaged to define the mean HU value of the lumbar spine. Sclerotic areas of the vertebral body related to degenerative changes were avoided when measuring HUs. Two independent reviewers performed these measurements. The reviewers were blinded to the individual's DEXA scores while determining the $\mathrm{HU}$ values.

\section{Statistical Analysis}

A sample size of 16 patients was estimated to provide 0.8 power for a correlation of $r=0.5$, with statistical significance defined as $p=0.05$. Patients were assessed for osteoporosis based on femoral neck DEXA-measured
TABLE 1. Mean scores from femoral neck DEXA, lumbar spine DEXA, and lumbar spine CT scanning in 48 patients with ASD

\begin{tabular}{ccccc}
\hline $\begin{array}{c}\text { Femoral } \\
\text { Neck } \\
\text { T-Score }\end{array}$ & $\begin{array}{c}\text { Femoral } \\
\text { Neck BMD } \\
\left(\mathrm{g} / \mathrm{cm}^{2}\right)\end{array}$ & $\begin{array}{c}\text { Lumbar } \\
\text { Spine } \\
\text { T-Score }\end{array}$ & $\begin{array}{c}\text { Lumbar } \\
\text { Spine BMD } \\
\left(\mathrm{g} / \mathrm{cm}^{2}\right)\end{array}$ & $\begin{array}{c}\text { Lumbar } \\
\text { CT HUs }\end{array}$ \\
\hline$-1.15 \pm 0.75$ & $0.73 \pm 0.10$ & $0.146 \pm 1.56$ & $1.06 \pm 0.17$ & $127.94 \pm 43.18$ \\
\hline
\end{tabular}

Values are presented as mean \pm SD.

BMD, lumbar spine DEXA-measured BMD, and lumbar spine CT-measured HUs. Osteoporosis was defined as a BMD of $<0.76 \mathrm{~g} / \mathrm{cm}^{2}$; osteopenia was defined as a BMD of 0.76 to $0.9 \mathrm{~g} / \mathrm{cm}^{2}$; and normal was considered to be a BMD of $>0.9 \mathrm{~g} / \mathrm{cm}^{2}$. For the lumbar CT scan, an HU < 112.4 was defined as osteoporosis; an HU between 112.4 and 150.1 was defined as osteopenia; and an HU > 150.1 was defined as normal. ${ }^{38}$ The number of patients with values consistent with osteoporosis was determined for each measure.

Pearson correlation coefficients were calculated between femoral neck BMD, lumbar spine BMD, and lumbar spine HUs. Predetermined classification of correlation strengths were used where $r>0.7$ was considered high agreement, $r>0.5$ was considered moderate agreement, and $\mathrm{r}<0.3$ was considered poor agreement. ${ }^{15}$ The chisquare test was used to compare counts of normal, osteopenia, and osteoporosis among the 3 measurements of BMD.

All statistical analyses were performed using IBM SPSS Statistics V23.

\section{Results}

One hundred fourteen eligible patients were identified and 48 had complete records available for analysis. The vast majority of patients were female $(n=43$ [89.6\%] of 48 ) and white ( $n=47$ [97.9\%] of 48), compared with nonwhite $(\mathrm{n}=1[2.1 \%])$. The mean age at the time of surgery was 62.1 years (range $36-83$ years).

Results from femoral neck DEXA, lumbar spine DEXA, and lumbar spine CT scans are shown in Table 1. The bone health status of patients, as defined by the different imaging modalities, is shown in Table 2. Femoral neck DEXA images were more likely to diagnose osteopenia than lumbar DEXA or lumbar CT HU measurements (femoral neck DEXA 26 [54.2\%]; lumbar DEXA 8 [17.4\%]; and lumbar CT scan 6 [12.5\%]).

Correlations between lumbar spine CT HU, lumbar spine DEXA, and femoral neck DEXA measurements are shown in Table 3. There was a low-moderate correlation between lumbar spine CT and lumbar spine DEXA measurements $(\rho=0.463, p<0.001)$, and there was poor correlation between lumbar spine CT and femoral neck DEXA measurements $(\rho=0.303, p=0.036)$.

\section{Discussion}

As the population ages the prevalence of ASD, and ASD surgeries, rises. There is mounting evidence that bone health is one of the most important factors related to 
TABLE 2. Bone health defined by imaging modality in 48 patients with ASD

\begin{tabular}{lccc}
\hline & \multicolumn{3}{c}{ No. of Patients (\%) } \\
\cline { 2 - 4 } Bone Health & $\begin{array}{c}\text { Femoral } \\
\text { Neck DEXA }\end{array}$ & $\begin{array}{c}\text { Lumbar } \\
\text { Spine DEXA }\end{array}$ & $\begin{array}{c}\text { Lumbar } \\
\text { CT }\end{array}$ \\
\hline Normal & $21(43.8)$ & $36(78.3)$ & $39(81.3)$ \\
\hline Osteopenia & $26(54.2)$ & $8(17.4)$ & $6(12.5)$ \\
\hline Osteoporosis & $1(2.1)$ & $2(4.3)$ & $3(6.2)$ \\
\hline
\end{tabular}

successful ASD surgery due to the relationship with perioperative complications. BMD is directly related to the ability of screws to resist pullout, and it predicts postoperative instability and fracture risk; low BMD may be a risk factor for developing pseudarthrosis..$^{12,17,21,30,35,36}$ However, despite increasing awareness of the importance of bone health in the success of spinal fusion, Wagner et al. found that in patients in whom osteoporosis was retrospectively diagnosed at the time of surgery, $64 \%$ had not undergone any preoperative evaluation or workup. ${ }^{38}$ Thus, there is a unique and mandatory opportunity to screen patients being considered for ASD surgery for the diagnoses of osteopenia and osteoporosis. ${ }^{11}$

There is debate as to whether DEXA is the best imaging modality for diagnosing osteoporosis. DEXA scans are $2 \mathrm{D}$ and measure the density-per-area (grams/square centimeter), but are unable to measure the volumetric density (grams/cubic centimeter) of bone. ${ }^{20}$ Additionally, degenerative changes and structures overlying the spine (e.g., aortic calcifications) lead to false elevations in lumbar DEXA measurements and false-negative results. ${ }^{20}$ Quantitative CT scanning has been proposed as an alternative modality for diagnosing osteoporosis. Advantages of CT over DEXA include true volumetric measurement of the bone, isolation of trabecular bone measurements (which are more sensitive to monitoring changes with disease), and better identification of fragility fractures. ${ }^{3,4,20,40}$ However, higher radiation doses and a lack of longitudinal data showing how CT predicts fracture risk have precluded its widespread clinical use.

Given the lumbar changes and morphological abnormalities in patients with ASD, femoral neck DEXA remains the most widely used modality for diagnosing osteoporosis in clinical practice. HUs on lumbar CT scans are an opportunistic screening tool in patients undergoing spine surgery, as they have shown strong correlations with BMD, T-scores, and vertebral compressive strength. ${ }^{19,32,33}$ Additionally, significantly higher HU measurements have been found in patients with successful lumbar interbody fusion, ${ }^{34}$ and significantly lower HU measurements have been observed in patients with adjacent-segment fracture after fusion. ${ }^{22}$

In the present study of ASD patients, we did not find lumbar CT HU measurements to be an effective screening tool for low BMD. The correlation between lumbar HUs and femoral neck BMD was poor $(r=0.303)$. Not surprisingly, the correlation between lumbar HUs and lumbar BMD was higher $(r=0.463)$. A greater number of patients were diagnosed with osteopenia when using the
TABLE 3. Pearson correlations among imaging modalities

\begin{tabular}{lccc}
\hline \multicolumn{1}{c}{ Modality } & $\begin{array}{c}\text { Lumbar } \\
\text { DEXA }\end{array}$ & $\begin{array}{c}\text { Femoral Neck } \\
\text { DEXA }\end{array}$ & $\begin{array}{c}\text { Lumbar } \\
\text { CT HUs }\end{array}$ \\
\hline Lumbar DXA & 1.00 & $0.298(p=0.045)$ & $0.463(p=0.001)$ \\
\hline Femoral neck DXA & - & 1.00 & $0.303(p=0.036)$ \\
\hline Lumbar CT HUs & - & - & 1.00 \\
\hline$-=$ not applicable & & &
\end{tabular}

femoral neck BMD measurements, compared with both lumbar HUs and BMD (femoral neck DEXA 26 [54.2\%]; lumbar DEXA 8 [17.4\%]; and lumbar CT HUs 6 [12.5\%]; $\mathrm{p}<0.001)$. Thus, lumbar CT HUs did not provide an accurate assessment of bone health, using the femoral neck as a reference, in this population of ASD patients.

Wagner et al. compared lumbar CT HUs to DEXA scans and established that patients with HUs of less than 150 should be referred for further evaluation and treatment of osteopenia or osteoporosis before surgery. ${ }^{38} \mathrm{How}-$ ever, in our spinal deformity population, using the cutoff of $150 \mathrm{HUs}$ would have identified $81.3 \%$ of our patients as having normal BMD, while femoral neck DEXA identified only $43.8 \%$ as having normal bone health. A similar number of false-negative results would be achieved using lumbar spine DEXA scans (75\% recorded normal BMD). Thus, relying solely on the lumbar CT HU measurements in our population would have missed an additional 37.5\% of patients who may have benefited from preoperative bone health evaluation and optimization.

While the opportunistic CT scan to assess for bone health is a convenient tool in patients undergoing spinal fusion surgery, it does not appear to be useful in an ASD population. This study reinforces the importance of utilizing established recommendations and protocols for evaluating and treating bone health abnormalities in this population. The National Osteoporosis Foundation recommends that DEXA scans be obtained in all women over 65 and in men over 70 years of age (regardless of clinical risk factors), in younger peri- and postmenopausal women, in men 50-69 with clinical risk factors for fracture, in adults who have had a fracture after the age of 50, and in adults with a condition (e.g., rheumatoid arthritis) or taking a medication (e.g., glucocorticoids) associated with low bone mass. ${ }^{9}$

Limitations of this study include the retrospective design. A substantial portion of the eligible patients did not have data for analysis. While the study is powered to answer the study question, selection bias may affect the availability of data and our cohort may not be representative of ASD patients. A consecutive series of ASD patients could offer improved, generalizable conclusions. Furthermore, our study population is largely white females, and, while this reflects most ASD practices, the conclusions may not be true for males or other races. DEXA scans may have been obtained in individuals for whom there was clinical concern only. While a limitation, this bias may have encouraged the diagnosis of osteopenia or osteoporosis, which was not the case. We have investigated only patients with lumbar ASD, and the findings here may not 
be consistent with those of other adult deformities, including iatrogenic deformity. The absence of a control group without spinal deformity may be seen as a limitation, but those correlations have previously been established, and we have followed previously published $\mathrm{HU}$ measurement techniques. ${ }^{33,38}$ Similarly, the choice of 150 HUs as a cutoff for "normal" is a diagnostic value; an "opportunistic screen" may require a lower threshold for diagnosing osteopenia/osteoporosis. Finally, the association between lumbar HU measurement and perioperative complications related to bone quality has not been assessed. A cohort of CT-diagnosed and DEXA-confirmed patients with osteoporosis may help elucidate the clinical utility of "opportunistic" BMD measurements.

\section{Conclusions}

While measuring HUs on lumbar CT scans can be an alternative method by which to assess BMD in patients undergoing spinal fusion surgery, it may not be an effective tool in the primary ASD population. Preoperative evaluation and optimization of bone health are essential aspects of ASD care. Surgeons should follow established protocols and National Osteoporosis Foundation recommendations as part of the preoperative care of patients in these complicated cases.

\section{References}

1. Abrahamsen B, van Staa T, Ariely R, Olson M, Cooper C: Excess mortality following hip fracture: a systematic epidemiological review. Osteoporos Int 20:1633-1650, 2009

2. Battié MC, Videman T: Lumbar disc degeneration: epidemiology and genetics. J Bone Joint Surg Am 88 (Suppl 2):3-9, 2006

3. Bergot C, Laval-Jeantet AM, Hutchinson K, Dautraix I, Caulin F, Genant HK: A comparison of spinal quantitative computed tomography with dual energy X-ray absorptiometry in European women with vertebral and nonvertebral fractures. Calcif Tissue Int 68:74-82, 2001

4. Black DM, Greenspan SL, Ensrud KE, Palermo L, McGowan JA, Lang TF, et al: The effects of parathyroid hormone and alendronate alone or in combination in postmenopausal osteoporosis. N Engl J Med 349:1207-1215, 2003

5. Blake GM, Fogelman I: Role of dual-energy X-ray absorptiometry in the diagnosis and treatment of osteoporosis. J Clin Densitom 10:102-110, 2007

6. Burge R, Dawson-Hughes B, Solomon DH, Wong JB, King A, Tosteson A: Incidence and economic burden of osteoporosis-related fractures in the United States, 2005-2025. J Bone Miner Res 22:465-475, 2007

7. Carter OD, Haynes SG: Prevalence rates for scoliosis in US adults: results from the first National Health and Nutrition Examination Survey. Int J Epidemiol 16:537-544, 1987

8. Colón-Emeric C, Kuchibhatla M, Pieper C, Hawkes W, Fredman L, Magaziner J, et al: The contribution of hip fracture to risk of subsequent fractures: data from two longitudinal studies. Osteoporos Int 14:879-883, 2003

9. Cosman F, de Beur SJ, LeBoff MS, Lewiecki EM, Tanner $\mathrm{B}$, Randall S, et al: Clinician's guide to prevention and treatment of osteoporosis. Osteoporos Int 25:2359-2381, 2014

10. Dalle Carbonare L, Giannini S, Sartori L, Nobile M, Ciuffreda M, Silva-Netto F, et al: Lumbar osteoarthritis, bone mineral density, and quantitative ultrasound. Aging (Milano) 12:360-365, 2000

11. Dipaola CP, Bible JE, Biswas D, Dipaola M, Grauer JN,
Rechtine GR: Survey of spine surgeons on attitudes regarding osteoporosis and osteomalacia screening and treatment for fractures, fusion surgery, and pseudoarthrosis. Spine J 9:537-544, 2009

12. Dvorak MF, Pitzen T, Zhu Q, Gordon JD, Fisher CG, Oxland TR: Anterior cervical plate fixation: a biomechanical study to evaluate the effects of plate design, endplate preparation, and bone mineral density. Spine (Phila Pa 1976) 30:294301, 2005

13. Gupta MC: Degenerative scoliosis. Options for surgical management. Orthop Clin North Am 34:269-279, 2003

14. Healey JH, Lane JM: Structural scoliosis in osteoporotic women. Clin Orthop Relat Res (195):216-223, 1985

15. Hinkle DE, Wiersma W, Jurs SG: Applied Statistics for the Behavioral Sciences. Boston: Houghton Mifflin, 1998

16. Jeon YK, Shin MJ, Shin YB, Kim CR, Kim SJ, Ko HY, et al: Effect of increased axial rotation angle on bone mineral density measurements of the lumbar spine. Spine J 14:2150 2154, 2014

17. Johnsson KE, Willner S, Johnsson K: Postoperative instability after decompression for lumbar spinal stenosis. Spine (Phila Pa 1976) 11:107-110, 1986

18. Kinoshita H, Tamaki T, Hashimoto T, Kasagi F: Factors influencing lumbar spine bone mineral density assessment by dual-energy X-ray absorptiometry: comparison with lumbar spinal radiogram. J Orthop Sci 3:3-9, 1998

19. Lee S, Chung CK, Oh SH, Park SB: Correlation between bone mineral density measured by dual-energy X-ray absorptiometry and Hounsfield units measured by diagnostic CT in lumbar spine. J Korean Neurosurg Soc 54:384-389, 2013

20. Link TM: Osteoporosis imaging: state of the art and advanced imaging. Radiology 263:3-17, 2012

21. Marshall D, Johnell O, Wedel H: Meta-analysis of how well measures of bone mineral density predict occurrence of osteoporotic fractures. BMJ 312:1254-1259, 1996

22. Meredith DS, Schreiber JJ, Taher F, Cammisa FP Jr, Girardi FP: Lower preoperative Hounsfield unit measurements are associated with adjacent segment fracture after spinal fusion. Spine (Phila Pa 1976) 38:415-418, 2013

23. Muraki S, Yamamoto S, Ishibashi H, Horiuchi T, Hosoi T, Orimo H, et al: Impact of degenerative spinal diseases on bone mineral density of the lumbar spine in elderly women. Osteoporos Int 15:724-728, 2004

24. Nilsonne U, Lundgren KD: Long-term prognosis in idiopathic scoliosis. Acta Orthop Scand 39:456-465, 1968

25. Paiva LC, Filardi S, Pinto-Neto AM, Samara A, Marques Neto JF: Impact of degenerative radiographic abnormalities and vertebral fractures on spinal bone density of women with osteoporosis. Sao Paulo Med J 120:9-12, 2002

26. Pappou IP, Girardi FP, Sandhu HS, Parvataneni HK, Cammisa FP Jr, Schneider R, et al: Discordantly high spinal bone mineral density values in patients with adult lumbar scoliosis. Spine (Phila Pa 1976) 31:1614-1620, 2006

27. Pérennou D, Marcelli C, Hérisson C, Simon L: Adult lumbar scoliosis. Epidemiologic aspects in a low-back pain population. Spine (Phila Pa 1976) 19:123-128, 1994

28. Rand T, Schneider B, Grampp S, Wunderbaldinger P, Migsits $\mathrm{H}$, Imhof $\mathrm{H}$ : Influence of osteophytic size on bone mineral density measured by dual X-ray absorptiometry. Acta Radiol 38:210-213, 1997

29. Reid IR, Evans MC, Ames R, Wattie DJ: The influence of osteophytes and aortic calcification on spinal mineral density in postmenopausal women. J Clin Endocrinol Metab 72:1372-1374, 1991

30. Reitman CA, Nguyen L, Fogel GR: Biomechanical evaluation of relationship of screw pullout strength, insertional torque, and bone mineral density in the cervical spine. J Spinal Disord Tech 17:306-311, 2004

31. Robin GC, Span Y, Steinberg R, Makin M, Menczel J: Scoli- 
osis in the elderly: a follow-up study. Spine (Phila Pa 1976) 7:355-359, 1982

32. Romme EA, Murchison JT, Phang KF, Jansen FH, Rutten EP, Wouters EF, et al: Bone attenuation on routine chest CT correlates with bone mineral density on DXA in patients with COPD. J Bone Miner Res 27:2338-2343, 2012

33. Schreiber JJ, Anderson PA, Rosas HG, Buchholz AL, Au AG: Hounsfield units for assessing bone mineral density and strength: a tool for osteoporosis management. J Bone Joint Surg Am 93:1057-1063, 2011

34. Schreiber JJ, Hughes AP, Taher F, Girardi FP: An association can be found between Hounsfield units and success of lumbar spine fusion. HSS J 10:25-29, 2014

35. Seller K, Wahl D, Wild A, Krauspe R, Schneider E, Linke B: Pullout strength of anterior spinal instrumentation: a product comparison of seven screws in calf vertebral bodies. Eur Spine J 16:1047-1054, 2007

36. Steinmann JC, Herkowitz HN: Pseudarthrosis of the spine. Clin Orthop Relat Res (284):80-90, 1992

37. U.S. Department of Health and Human Services: Bone Health and Osteoporosis: A Report of the Surgeon General. Rockville, MD: U.S. Department of Health and Human Services, Office of the Surgeon General, 2004

38. Wagner SC, Formby PM, Helgeson MD, Kang DG: Diagnosing the undiagnosed: osteoporosis in patients undergoing lumbar fusion. Spine (Phila Pa 1976) 41:E1279-E1283, 2016

39. Wright NC, Looker AC, Saag KG, Curtis JR, Delzell ES,
Randall S, et al: The recent prevalence of osteoporosis and low bone mass in the United States based on bone mineral density at the femoral neck or lumbar spine. J Bone Miner Res 29:2520-2526, 2014

40. Yu W, Glüer CC, Grampp S, Jergas M, Fuerst T, Wu CY, et al: Spinal bone mineral assessment in postmenopausal women: a comparison between dual X-ray absorptiometry and quantitative computed tomography. Osteoporos Int 5:433-439, 1995

\section{Disclosures}

Dr. Hershman is a consultant for DePuy Synthes.

\section{Author Contributions}

Conception and design: Kelly, Kang. Acquisition of data: Kohan, Nemani. Analysis and interpretation of data: Kelly, Hershman, Kang. Drafting the article: Kelly, Kohan. Critically revising the article: Kelly, Kohan, Hershman. Reviewed submitted version of manuscript: all authors. Approved the final version of the manuscript on behalf of all authors: Kelly. Statistical analysis: Kelly.

\section{Correspondence}

Michael P. Kelly, Department of Orthopedic Surgery, Washington University in St. Louis, 660 S Euclid Ave., Campus Box 8233, St. Louis, MO 63110. email: kellymi@wustl.edu. 